Monika Szturmowicz', Izabela Siemion-Szcześniak', Dorota Wyrostkiewicz', Magdalena Klatt², Sylwia Brzezińska ${ }^{2}$, Anna Zabost ${ }^{2}$, Anna Lewandowska', Dorota Filipczak ${ }^{2}$, Karina Oniszh ${ }^{3}$, Agnieszka Skoczylas ${ }^{4}$, Ewa Augustynowicz-Kopeć ${ }^{2}$, Jan Kuś

${ }^{1} 1^{\text {st }}$ Department of Lung Diseases, National Tuberculosis and Lung Diseases Research Institute, Warsaw, Poland

${ }^{2}$ Departmentof Microbiology National Tuberculosis and Lung Diseases Research Institute, Warsaw, Poland

${ }^{3}$ Department of Radiology, National Tuberculosis and Lung Diseases Research Institute, Warsaw, Poland

${ }^{4}$ Geriatrics Department, National Institute of Geriatrics, Rheumatology and Rehabilitation, Warsaw, Poland

\title{
Factors predisposing to non-tuberculous mycobacterial lung disease in the patients with respiratory isolates of non-tuberculous mycobacteria
}

The authors declare no financial disclosure

\begin{abstract}
Introduction: An increasing incidence rate of respiratory isolates of non-tuberculous mycobacteria (NTM) has been noted recently in most European countries as well as in the US. Despite many publications, there is no consensus concerning the importance of different factors in promoting NTM lung disease (NTMLD).

The aim of the present retrospective study was to analyse patients with positive NTM respiratory isolates in search of factors predisposing to NTMLD.

Material and methods: 73 patients, 23 males, 50 females, median age 62.2 years, in whom NTM have been cultured from respiratory specimen (sputum and/or bronchial washings), in the period 2010-2015, entered the study.

Results: NTMLD (according to ATS/IDSA) has been recognised in 36 patients, airways colonisation by NTM — in 37 patients. NTMLD was diagnosed more often in the patients infected with $M$. kansasii, $M$. abscessus and $M$. avium/ $M$. intracellulare comparing to those infected with $M$. xenopi, $M$. gordonae and $M$. fortuitum ( $\mathrm{p}<0.0001$ ). The proportion of females to males was significantly higher in the NTMLD group comparing to the colonisation group $(\mathrm{p}<0.007)$. Previous tuberculosis or mycobacteriosis were noted significantly more frequently in the group of patients with NTMLD comparing to the colonisation group ( $28 \%$ vs $8 \%, p=0.038)$. Univariate regression analysis revealed $M$. kansasii, female gender, and previous tuberculosis or mycobacteriosis as significant predictors of NTMLD. Conclusions: The risk factors of NTMLD recognition in the presented group of patients were the following: female gender, M. kansasii isolation, as well as past tuberculosis or mycobacteriosis.
\end{abstract}

Key words: non-tuberculous mycobacteria, COPD, bronchiectasis, interstitial lung disease, corticosteroids

Adv Respir Med. 2018; 86: 261-267

\section{Introduction}

Non-tuberculous mycobacteria (NTM) are environmental organisms, present in natural and artificial water sources as well as in dust and in soil $[1,2]$. They may colonise human bronchial, gastrointestinal and urinary tracts, causing no symptoms of the disease, never- theless, in some individuals NTM may be pathogenic [3].

The increasing incidence rate of respiratory isolates of NTM as well as NTM caused lung disease (NTMLD) has been noted recently in most European countries as well as in the US [4-8]. This concerns especially the increase of $M$. avium, $M$. fortuitum and $M$. abscessus $[4,5,8,9]$. The

Address for correspondence: Monika Szturmowicz, $1^{\text {st }}$ Department of Lung Diseases, National Tuberculosis and Lung Diseases Research Institute, Warsaw, Poland,

e-mail: monika.szturmowicz@gmail.com

DOI: 10.5603/ARM.a2018.0043

Received: 10.09 .2018

Copyright (C) 2018 PTChP

ISSN 2451-4934 
growing incidence of NTM caused lung disease is probably brought about by an increasing number of patients with risk factors, such as chronic lung diseases, immunosuppressive treatment as well as aging of the population [4, 8, 10-12].

NTM isolation from a respiratory specimen is not an unambiguous sign of NTMLD. The criteria of NTMLD are defined in the ATS/IDSA recommendations from 2007 [13] and the latest BTS recommendations [14]. The proportion of patients with positive respiratory isolates of NTM, in whom NTMLD is diagnosed depends on the pathogenic characteristics of NTM and clinical characteristics of the population [15]. Despite many publications, there is no consensus in the literature concerning the importance of different factors in promoting NTMLD development.

Thus, the aim of the present retrospective study was to analyse the clinical and microbiological data in search of factors predisposing to NTMLD in the population of patients with positive NTM respiratory isolates.

\section{Material and methods}

\section{Population}

73 patients, 23 males, 50 females, median age 62.2 years ( $20-85$ years), in whom non-tuberculous mycobacteria (NTM) have been cultured from respiratory specimens (sputum and/or bronchial washings), in the period from 2010 to 2015, entered the study. The study approval was obtained from Ethics Committee (no 9/2015).

\section{Diagnostic criteria}

The criteria of the recognition of non-tuberculous mycobacterial lung disease (NTMLD) have been applied according to the ATS/IDSA recommendations (Table 1) [13]. The factors potentially influencing development of NTMLD, such as: age, gender, body mass index (BMI), smoking habits, comorbidities and their treatment, were analysed.

\section{NTM cultures and identification}

The respiratory specimens (sputum and/or bronchial washings) were decontaminated with the sodium hydroxide and N-acetyl-L-cysteine (NaOH/NALC) method. The strains were cultured on solid media: egg-based Lowenstein-Jensen medium, Stonebrink medium and in automated system MGIT (Becton Dickinson).

Identification of culture was performed using the molecular test GenoType CM (Hain Lifescience), version 1.0 and 2.0. The procedure for identifying strains consists of three steps: isolation of DNA, amplification using primers labeled with biotin and a reverse hybridisation. The GenoType CM test using the DNA-STRIP method allows the identification of Mycobacterium tuberculosis complex strains and 14 clinically relevant NTM within a single procedure.

\section{Statistical methods}

Data were analysed in $\mathrm{R}$ environment [16]. The differences between the groups concerning the distribution of categorical variables were assessed with chi-square test. Wilcoxon rank sum test with continuity correction was used for the comparison of continuous variables in two different groups. Univariate logistic regression concerning prediction of NTMLD was performed and odds ratios with its $95 \%$ confidence interval were calculated, $\mathrm{p}$-value $<0.05$ was considered as statistically significant.

\section{Results}

NTMLD has been recognised in 36 patients, airways colonisation by NTM - in 37 patients. Demographic data and smoking habits in the group of NTMLD versus the group with NTM colonisation are presented in Table 2 .

The proportion of females to males was significantly higher in the NTMLD group comparing to the colonisation group. A tendency towards lower age and BMI was noted in the NTMLD group comparing to the colonisation group.

\section{Table 1. Principles of recognition of NTMLD according to ATS/IDSA [13]}

\begin{tabular}{ll}
\hline Clinical & 1.Pulmonary symptoms, nodular or cavitary opacities on chest radiograph, or a high-resolution CT scan that shows mul- \\
tifocal bronchiectasis with multiple small nodules \\
and \\
2. Appropriate exclusion of other diagnoses \\
Microbiologic & 1. Positive culture results from at least two separate expectorated sputum samples \\
or & 2. Positive culture results from at least one bronchial wash or lavage \\
or & 3.Transbronchial or other lung biopsy with mycobacterial histopathological features (granulomatous inflammation or \\
& acid fast bacilli) and positive culture for NTM of: lung specimen, bronchial wash or sputum (at least one)
\end{tabular}


Table 2. Demographic data and smoking habits according to final diagnosis in $\mathbf{7 3}$ patients with positive respiratory isolates for non-tuberculous mycobacteria (NTM)

\begin{tabular}{|c|c|c|c|c|c|}
\hline Diagnosis & No of pts & $\begin{array}{c}\text { Age (years. median, } \\
\text { range) }\end{array}$ & Males/females & $\begin{array}{c}\text { Smokers } \\
\text { No }(\%)\end{array}$ & $\begin{array}{c}\mathrm{BMI}\left(\mathrm{kg} / \mathrm{m}^{2}\right) \text {, } \\
\text { mean, SD }\end{array}$ \\
\hline NTMLD & 36 & $\begin{array}{c}58.5 \\
(20-85)\end{array}$ & $6 / 30^{*}$ & $16(44)$ & $\begin{array}{c}22.7 \\
( \pm 4.74)\end{array}$ \\
\hline NTM colonisation & 37 & $\begin{array}{c}66.2 \\
(20-78)\end{array}$ & $17 / 20^{*}$ & $17(46)$ & $\begin{array}{c}24.4 \\
( \pm 5.04)\end{array}$ \\
\hline Total & 73 & $\begin{array}{c}62.2 \\
(20-85)\end{array}$ & $23 / 50$ & $33(45)$ & $\begin{array}{c}23.7 \\
( \pm 4.9)\end{array}$ \\
\hline
\end{tabular}

${ }^{*} p=0.007$; NTMLD: non-tuberculous mycobacterial lung disease; BMI: body mass index; SD: standard deviation

Table 3. Distribution of NTM species according to final diagnosis in 66 patients with positive respiratory isolates for single non-tuberculous mycobacteria (NTM)

\begin{tabular}{|c|c|c|c|c|c|c|c|c|}
\hline \multirow[t]{2}{*}{ Diagnosis } & \multirow{2}{*}{$\begin{array}{c}\text { No of } \\
\text { pts }\end{array}$} & \multicolumn{7}{|c|}{ NTM species } \\
\hline & & $\begin{array}{c}M . \\
\text { kansasii }\end{array}$ & M. avium & M. intracel. & $\begin{array}{c}M . \\
\text { abscessus }\end{array}$ & $\begin{array}{c}M . \\
\text { gordonae }\end{array}$ & $\begin{array}{c}M . \\
\text { fortuitum }\end{array}$ & $\begin{array}{c}M . \\
\text { xenop }\end{array}$ \\
\hline NTMLD & 33 & 13 & 10 & 4 & 2 & 1 & 0 & 3 \\
\hline $\begin{array}{l}\text { NTM } \\
\text { colonisation }\end{array}$ & 33 & 3 & 6 & 2 & 0 & 13 & 4 & 5 \\
\hline Total & 66 & 16 & 16 & 6 & 2 & 14 & 4 & 8 \\
\hline
\end{tabular}

The NTM species identification revealed: M. kansasii - in 16 patients (22\%), M. avium - in 16 (22\%), M. gordonae - in 14 (19\%), M. xenopi - in $8(11 \%), M$. intracellulare - in $6(8 \%)$, M. fortuitum - in 4 (6\%), M. abscessus - in $2(3 \%)$, more than one type of mycobacteria - in 7 patients (10\%). The distribution of NTM species in the NTMLD group comparing to the colonisation group, was performed in 66 patients with a single respiratory NTM isolate (Table 3).

NTMLD has been diagnosed in both individuals infected with M.abscessus, in $81 \%$ of patients infected - with M.kansasii, 64\% of subjects infected with $M$. avium/M. intracellulare, $38 \%$ of those with $M$. xenopi, $7 \%$ of infected with $M$. gordonae and none - with $M$. fortuitum. The proportion of NTMLD among patients with more pathogenic NTM types (M. abscessus, $M$. kansasii and M. avium/M. intracellulare) and those less pathogenic ( $M$. xenopi, M. gordone, M. fortuitum) - were 29/40 (73\%) and 4/26 (15\%), respectively, $\mathrm{p}<0.0001$.

In majority of patients - 70/73 (96\%), coexisting diseases that could predispose to NTMLD were noted: in 25/73 (34\%) - one, in 26/73(36\%) — two, and in 19/73(26\%) - more than two diseases. Analysis of coexisting disorders is presented in table 4. Most frequent comorbidities were: bronchiectasis - in 24 patients - 33\%, chronic obstructive pulmonary disease (COPD) - in 18 individuals $(25 \%)$ and interstitial lung diseases (ILD) - in 18 subjects (25\%): lung fibrosis due to collagen tissue disease (4 cases), cryptogenic organising pneumonia (3), sarcoidosis (3), hypersensitivity pneumonitis (2), Langerhans cell histiocytosis (1), NSIP (1), lung fibrosis not classified (4). Oral corticosteroids (CS) were used by 17 (23\%) patients, in 10 - as the only therapy, in 7 - combined with immunosuppressive therapy. The median prednisone dose was $10 \mathrm{mg} /$ day (range 5-40 mg). Immunosuppressive drugs were used by 9 (12\%) patients: azathioprine 100-150 $\mathrm{mg} /$ day - by 4 , methotrexate $10 \mathrm{mg} /$ week -1 , rituximab -1 , hydroxychloroquine -1 , and systemic chemotherapy due to lung cancer - by 2 individuals.

The influence of various pre-existing diseases on NTMLD development has been investigated (Table 4). Previous tuberculosis or mycobacteriosis were noted significantly more frequently in the group of patients with NTMLD comparing to the colonisation group (28\% and $8 \%$, respectively, $\mathrm{p}=0.038$ ). COPD was diagnosed more often in the colonisation group comparing to NTMLD ( $30 \%$ and $19 \%$ respectively), nevertheless, the differences were not significant. 
Table 4. Coexisting diseases and their treatment according to final diagnosis in $\mathbf{7 3}$ patients with positive respiratory isolates for non-tuberculous mycobacteria (NTM)

\begin{tabular}{|c|c|c|c|c|}
\hline $\begin{array}{l}\text { Coexisting } \\
\text { disease }\end{array}$ & $\begin{array}{c}\text { NTMLD } \\
36 \text { pts } \\
\text { no }(\%)\end{array}$ & $\begin{array}{c}\text { Colonisation } \\
37 \text { pts } \\
\text { no }(\%)\end{array}$ & $\begin{array}{c}\text { Total } \\
73 \text { pts } \\
\text { no }(\%)\end{array}$ & $\mathbf{p}$ \\
\hline Bronchiectasis & $12(33)$ & $12(33)$ & $24(33)$ & 0.94 \\
\hline COPD & $7(19)$ & $11(30)$ & $18(25)$ & 0.25 \\
\hline Astma/ABPA & $2(6)$ & $5(14)$ & $7(10)$ & 0.24 \\
\hline ILD & $10(28)$ & $8(22)$ & $18(25)$ & 0.63 \\
\hline CTD & $5(14)$ & $5(14)$ & $10(14)$ & 1.0 \\
\hline $\mathrm{CF}$ & $3(8)$ & $1(3)$ & $4(6)$ & 0.63 \\
\hline NPL & $9(25)$ & $7(19)$ & $16(22)$ & 0.62 \\
\hline Diabetes & $4(11)$ & $9(24)$ & $13(18)$ & 0.19 \\
\hline Hypothyreosis & $8(22)$ & $6(16)$ & $14(19)$ & 0.59 \\
\hline Previous tbc/myco & $10(28)$ & $3(8)$ & $13(18)$ & 0.038 \\
\hline None & $2(6)$ & $1(3)$ & $3(4)$ & 0.52 \\
\hline Systemic glucocorticoids & $10(28)$ & $7(19)$ & $17(23)$ & 0.53 \\
\hline $\begin{array}{l}\text { Immunosuppressive } \\
\text { drugs }\end{array}$ & $4(11)$ & $5(14)$ & $9(12)$ & 0.97 \\
\hline
\end{tabular}

Table 5. Factors significantly influencing NTMLD recognition, univariate regression analysis

\begin{tabular}{lccc}
\hline \multicolumn{4}{c}{ Univariate regression analysis } \\
\hline Factor & OR & $\mathbf{9 5 \% C l}$ & $\mathbf{p}$ \\
\hline M. kansasii & 4.87 & $1.42-16.72$ & 0.012 \\
M. gordonae & 0.044 & $0.005-0.36$ & 0.003 \\
Female gender & 3.69 & $1.23-11.05$ & 0.02 \\
Age & 0.96 & $0.927-0.999$ & 0.041 \\
BMI & 0.894 & $0.805-0.992$ & 0.035 \\
Previous TBC, NTMLD & 4.074 & $1.018-16.306$ & 0.047 \\
\hline
\end{tabular}

OR: odds ratio; $\mathrm{Cl}$ : confidence interval; $\mathrm{BMI}$ : body mass index; TBC: tuberculosis; NTMLD: non-tuberculous mycobacterial lung disease

The proportions of ILD and collagen tissue disease (CTD) in the groups of NTMLD and colonisation were equal. In the patients with NTMLD comparing to colonisation, the percentages of individuals treated with corticosteroids alone or in combination with immunosuppressive therapy were: $28 \%$ and $19 \%$ respectively, with the difference being nonsignificant.

Univariate analysis of factors possibly influencing NTMLD development is presented in table 5. The elements significantly increasing probability of NTMLD were: $M$. kansasii isolate, female gender, and previous tuberculosis or mycobacteriosis. The risk of NTMLD was lower in those diagnosed with $M$. gordonae, older ones and those with higher BMI.

\section{Discussion}

The analysis of factors predisposing to NTMLD development in our material revealed that women were more susceptible than men. Female gender was confirmed as a risk factor of NTMLD in univariate analysis.

The published data concerning this subject are discrepant. Adzic et al. [6] documented that $66 \%$ of NTMLD cases registered between 2009 and 2016 in the University Hospital for Pulmonology in Belgrade involved men. US epidemiological studies concerning NTM respiratory isolates registered by Integrated Health Care Delivery Systems (2004-2006) found females' predominance $[11,17,18]$. It seems that males predominate in the group of patients with NTMLD and underlying COPD [6, 19], whereas females - in the category of bronchiectatic form of NTMLD [15, 17]. Especially, the nodular bronchiectatic type of disease observed in non-smoking, thin and tall, 
postmenopausal women, with pectus excavatum, and mitral valve prolapse (MVP), called Lady Windermere syndrome, may be responsible for female predominance in some published series $[3,20]$. In our study, 3 non-smoking, postmenopausal women, with BMI 19.7-23.6 were identified, nevertheless, without MVP or pectus excavatum.

The microbiological analysis of our group of patients revealed that the most pathogenic among NTM species was M. kansasii (NTMLD developed in $81 \%$ of infected patients), and it was found to be an independent risk factor of NTMLD diagnosis. Another study coming from Poland confirmed high virulence of $M$. kansasii reporting clinically relevant lung disease in $82 \%$ of infected patients [21].

M. abscessus was identified by us in two patients only, nevertheless, both presented with NTMLD. Recent US report demonstrated the increase of NTM species belonging to $M$. abscessus group between 1994 and 2014 by 322\% [4]. The current proportion of NTMLD caused by $M$. $a b$ scessus is 20\% in Europe and 15\% in Japan [22].

NTM belonging to M. avium/M. intracellulare complex (MAC) were less pathogenic in our material, nevertheless, in $64 \%$ of the patients infected with these strains NTMLD has been recognised. Although MAC is less pathogenic than M. kansasii, it is the cause of most cases of lung disease in the US, Europe and Japan, due to a high rate of identification, especially in the aging population $[17,22,23]$.

The present study revealed high frequency of coexisting diseases that could predispose to NTM colonisation. $96 \%$ of patients had at least one illness diagnosed.

Among chronic lung diseases, the most frequent were COPD and bronchiectasis diagnosed in $25 \%$ and $33 \%$ of patients, respectively.

The relation between COPD and NTMLD has been the subject of many studies published recently. Hoefsloot et al. [24] found a $22 \%$ prevalence of NTM isolation in 73 consecutive patients with COPD exacerbation, the subjects with positive culture were significantly older than the others. Char et al. [25] confirmed the presence of necrotising granulomas in $10 \%$ of COPD patients, in whom lung volume reduction surgery has been performed.

The increased risk of NTMLD in COPD subjects may be partly associated with inhaled steroids use and dosage [26, 27]. Diel et al. [28] found an increased mortality rate among COPD individuals infected with NTM, comparing to those not infected.
Up to $30 \%$ of COPD patients are diagnosed with COPD-bronchiectasis overlap syndrome [29]. Gatheral et al. [30] found radiographic presence of bronchiectasis in $69 \%$ of COPD patients admitted with first exacerbation. The presence of bronchiectasis in COPD independently determined sputum isolation of $P$. aeruginosa and NTM [30] and correlated with increased frequency of exacerbations and mortality [31].

Recently published population survey from Israel revealed the presence of positive respiratory isolates for NTM in 105/6274 (1.7\%) patients with bronchiectasis [32]. The authors indicated female gender, age below 65 years, and antibiotic or prednisolone therapy as risk factors for NTM colonisation [32]. A multicentre, epidemiological study, conducted in the US, concerned the possible risk factors of NTMLD in two national samples of bronchiectasis patients [33]. Inhaled corticosteroids use have been reported by $30-40 \%$ of patients, in $60 \%$ of them for the time longer than 1 year [33]. These data indicate that further research is needed to clarify the safety of inhaled corticosteroids in the patients with bronchiectasis.

ILD have been noted in 25\% of our group with positive NTM isolates. Various types of ILD have been recognised. NTM have been cultured in those with prolonged course of disease, lung fibrosis was present in most cases. This was true also for both patients with hypersensitivity pneumonitis, thus the positive NTM isolates were regarded as colonisation in the course of previously diagnosed lung disease and not the allergy to NTM antigens.

The data concerning the risk of NTM infection in ILD are scarce. Axson et al. [34] found ILD in $6 \%$ of patients with positive NTM culture in UK primary care centres. Ito et al. [35] documented ILD in $6.6 \%$ of subjects with pulmonary MAC-related disease. Park et al. [36] found NTMLD in $2 \%$ of patients with idiopathic pulmonary fibrosis, majority of them have not been treated with immunosuppressive drugs.

In our material, the percentage of individuals receiving oral corticosteroids due to ILD or CTD was larger in NTMLD comparing to the observation group ( $28 \%$ and $19 \%$, respectively), nevertheless, the difference was not significant. The influence of immunosuppressive therapy on NTMLD development was difficult to assess, as in most patients it was combined with corticosteroids.

Brode et al. [37] noted an increased risk of NTM-related disease in the patients with rheumatoid arthritis receiving anti-TNF therapy, but also in those treated with corticosteroids in the dose 
exceeding $20 \mathrm{mg} /$ day or hydroxychloroquine. Our patients who developed NTMLD received prednisone in the median dose of $10 \mathrm{mg}$ per day.

NTMLD, in our study, was significantly more probable in patients with previous history of tuberculosis or mycobacteriosis than in the remaining ones. This is an important observation, as substantial proportion of NTMLD in Poland, as well as in Serbia, develop in the individuals with past tuberculosis [6, 21, 38]. It is not known, whether those patients represent the genetic predisposition to mycobacterial lung diseases or whether post-tuberculous parenchymal abnormalities predispose to colonisation by NTM and further NTMLD.

Our group of patients with positive NTM respiratory specimens also included 4 individuals with cystic fibrosis (CF). In 3 of them NTMLD developed, in 1 - colonisation was recognised.

Growing evidence exists in the literature that CF is a risk factor for NTMLD [39-41]. The study concerning $16153 \mathrm{CF}$ patients above 12 years of age, conducted by Adjemian et al. [40] revealed at least one NTM isolation in between 2010 and 2014 in $20 \%$ of them. The growing prevalence of NTM isolates was noted: from $11 \%$ in 2010 to $13.4 \%$ in 2014 [40]. Another study concerning $5333 \mathrm{CF}$ children and young adults below 16 years of age, indicated the increasing prevalence of positive cultures for NTM from $1.3 \%$ in 2010 to $3.8 \%$ in 2015 [41]. The types of NTM predominating both in children and in adults diagnosed with CF, were M. avium/M. intracellulare and M. abscessus $[40,41]$. In our group of CF patients, the NTM identification revealed: M. abscessus - in 1, M. intracellulare - in 2, and M.avium - in 1 person.

In conclusion, the presented analysis of patients with positive NTM respiratory isolates showed that factors significantly increasing probability of NTMLD recognition were the following: female gender, M. kansasii respiratory isolate, or past tuberculosis or mycobacteriosis. M. gordonae isolate, as well as higher age and BMI were combined with a significantly lower risk of NTMLD development.

\section{Conflict of interest}

The authors declare no conflict of interest.

\section{References:}

1. Honda JR, Alper S, Bai X, et al. Acquired and genetic host susceptibility factors and microbial pathogenic factors that predispose to nontuberculous mycobacterial infections. Curr Opin Immunol. 2018; 54: 66-73, doi: 10.1016/j. coi.2018.06.001, indexed in Pubmed: 29936307.
2. Johnson MM, Odell JA. Nontuberculous mycobacterial pulmonary infections. J Thorac Dis. 2014; 6(3): 210-220, doi: 10.3978/j. issn.2072-1439.2013.12.24, indexed in Pubmed: 24624285.

3. Field SK, Cowie RL. Lung disease due to the more common nontuberculous mycobacteria. Chest. 2006; 129(6): 1653-1672, doi: 10.1378/chest.129.6.1653, indexed in Pubmed: 16778288.

4. Donohue MJ. Increasing nontuberculous mycobacteria reporting rates and species diversity identified in clinical laboratory reports. BMC Infect Dis. 2018; 18(1): 163, doi: 10.1186/s12879018-3043-7, indexed in Pubmed: 29631541.

5. Shah NM, Davidson JA, Anderson LF, et al. Pulmonary Mycobacterium avium-intracellulare is the main driver of the rise in non-tuberculous mycobacteria incidence in England, Wales and Northern Ireland, 2007-2012. BMC Infect Dis. 2016; 16: 195, doi: 10.1186/s12879-016-1521-3, indexed in Pubmed: 27154015

6. Adzic-Vukicevic T, Barac A, Blanka-Protic A, et al. Clinical features of infection caused by non-tuberculous mycobacteria: 7 years' experience. Infection. 2018; 46(3): 357-363, doi: 10.1007/s15010-018-1128-2, indexed in Pubmed: 29476412.

7. Prevots DR, Loddenkemper R, Sotgiu G, et al. Nontuberculous mycobacterial pulmonary disease: an increasing burden with substantial costs. Eur Respir J. 2017; 49(4), doi: 10.1183/13993003.00374-2017, indexed in Pubmed: 28446563.

8. van Ingen J, Hoefsloot W, Dekhuijzen PNR, et al. The changing pattern of clinical Mycobacterium avium isolation in the Netherlands. Int J Tuberc Lung Dis. 2010; 14(9): 1176-1180, indexed in Pubmed: 20819265.

9. Hoefsloot W, van Ingen J, Andrejak C, et al. Nontuberculous Mycobacteria Network European Trials Group. The geographic diversity of nontuberculous mycobacteria isolated from pulmonary samples: an NTM-NET collaborative study. Eur Respir J. 2013; 42(6): 1604-1613, doi: 10.1183/09031936.00149212, indexed in Pubmed: 23598956.

10. Stout JE, Koh WJ, Yew WW. Update on pulmonary disease due to non-tuberculous mycobacteria. Int J Infect Dis. 2016; 45 123-134, doi: 10.1016/j.ijid.2016.03.006, indexed in Pubmed: 26976549.

11. Prevots DR, Shaw PA, Strickland D, et al. Nontuberculous mycobacterial lung disease prevalence at four integrated health care delivery systems. Am J Respir Crit Care Med. 2010; 182(7): 970-976, doi: 10.1164/rccm.201002-03100C, indexed in Pubmed: 20538958.

12. Wilińska E, Oniszh K, Augustynowicz-Kopeć E, et al. Non-tuberculous mycobacterial lung disease (NT MLD ) in patients with chronic thromboembolic pulmonary hypertension and idiopathic pulmonary arterial hypertension. Pneumonol Alergol Pol. 2014; 82(6): 495-502, doi: 10.5603/PiAP.2014.0066, indexed in Pubmed: 25339559.

13. Griffith DE, Aksamit T, Brown-Elliott BA, et al. ATS Mycobacterial Diseases Subcommittee, American Thoracic Society, Infectious Disease Society of America. An official ATS/IDSA statement: diagnosis, treatment, and prevention of nontuberculous mycobacterial diseases. Am J Respir Crit Care Med. 2007; 175(4): 367-416, doi: 10.1164/rccm.200604-571ST, indexed in Pubmed: 17277290.

14. Haworth CS, Banks J, Capstick T, et al. British Thoracic Society guidelines for the management of non-tuberculous mycobacterial pulmonary disease (NTM-PD). Thorax. 2017; 72(Suppl 2): ii1-ii64, doi: 10.1136/thoraxjnl-2017-210927, indexed in Pubmed: 29054853.

15. Glassroth J. Pulmonary disease due to nontuberculous mycobacteria. Chest. 2008; 133(1): 243-251, doi: 10.1378/ chest.07-0358, indexed in Pubmed: 18187749.

16. $\mathrm{R}$ core team (2018). R: A language and environment for statistical computing. R Foundation for Statistical Computing, Vienna, Austria. https://www.R-project.org/.

17. Winthrop KL, McNelley E, Kendall B, et al. Pulmonary nontuberculous mycobacterial disease prevalence and clinical features: an emerging public health disease. Am J Respir Crit Care Med. 2010; 182(7): 977-982, doi: 10.1164/rccm. 201003-0503OC, indexed in Pubmed: 20508209.

18. Adjemian J, Olivier KN, Seitz AE, et al. Prevalence of nontuberculous mycobacterial lung disease in U.S. Medicare beneficiaries. Am J Respir Crit Care Med. 2012; 185(8): 881-886, doi: 10.1164/rccm.201111-2016OC, indexed in Pubmed: 22312016. 
19. van Ingen J, Bendien SA, de Lange WCM, et al. Clinical relevance of non-tuberculous mycobacteria isolated in the Nijmegen-Arnhem region, The Netherlands. Thorax. 2009; 64(6): 502-506, doi: 10.1136/thx.2008.110957, indexed in Pubmed: 19213773.

20. Kim RD, Greenberg DE, Ehrmantraut ME, et al. Pulmonary nontuberculous mycobacterial disease: prospective study of a distinct preexisting syndrome. Am J Respir Crit Care Med. 2008; 178(10): 1066-1074, doi: 10.1164/rccm.200805-686OC, indexed in Pubmed: 18703788.

21. Bakuła Z, Kościuch J, Safianowska A, et al. Clinical, radiological and molecular features of Mycobacterium kansasii pulmonary disease. Respir Med. 2018; 139: 91-100, doi: 10.1016/j. rmed.2018.05.007, indexed in Pubmed: 29858008.

22. van Ingen J, Wagner D, Gallagher J, et al. NTM-NET. Poor adherence to management guidelines in nontuberculous mycobacterial pulmonary diseases. Eur Respir J. 2017; 49(2), doi: 10.1183/13993003.01855-2016, indexed in Pubmed: 28182571.

23. Suzuki K, Kurashima A, Tatsuno K, et al. Clinical significance and epidemiologic analyses of Mycobacterium avium and Mycobacterium intracellulare lung disease from post-marketing surveillance. Respir Investig. 2018; 56(1): 87-93, doi 10.1016/j.resinv.2017.11.001, indexed in Pubmed: 29325687.

24. Hoefsloot W, van Ingen J, Magis-Escurra C, et al. Prevalence of nontuberculous mycobacteria in COPD patients with exacerbations. J Infect. 2013; 66(6): 542-545, doi: 10.1016/j. jinf.2012.12.011, indexed in Pubmed: 23298891.

25. Char A, Hopkinson NS, Hansell DM, et al. Evidence of mycobacterial disease in COPD patients with lung volume reduction surgery; the importance of histological assessment of specimens: a cohort study. BMC Pulm Med. 2014; 14: 124, doi: 10.1186/1471-2466-14-124, indexed in Pubmed: 25086862.

26. Andréjak C, Nielsen R, Thomsen VØ, et al. Chronic respiratory disease, inhaled corticosteroids and risk of non-tuberculous mycobacteriosis. Thorax. 2013; 68(3): 256-262, doi: 10.1136/ thoraxjnl-2012-201772, indexed in Pubmed: 22781123.

27. Brode SK, Campitelli MA, Kwong JC, et al. The risk of mycobacterial infections associated with inhaled corticosteroid use. Eur Respir J. 2017; 50(3), doi: 10.1183/13993003.00037-2017, indexed in Pubmed: 28931659.

28. Diel R, Jacob J, Lampenius N, et al. Burden of non-tuberculous mycobacterial pulmonary disease in Germany. Eur Respir J. 2017; 49(4), doi: 10.1183/13993003.02109-2016, indexed in Pubmed: 28446559.

29. Hurst JR, Elborn JS, De Soyza A, et al. BRONCH-UK Consortium. COPD-bronchiectasis overlap syndrome. Eur Respir J. 2015; 45(2): 310-313, doi: 10.1183/09031936.00170014, indexed in Pubmed: 25653262.

30. Gatheral T, Kumar N, Sansom B, et al. COPD-related bronchiectasis; independent impact on disease course and outcomes. COPD. 2014; 11(6): 605-614, doi:
10.3109/15412555.2014.922174, indexed in Pubmed: 24983298.

31. Martínez-García MA, de la Rosa Carrillo D, Soler-Cataluña JJ, et al. Prognostic value of bronchiectasis in patients with moderate-to-severe chronic obstructive pulmonary disease. Am J Respir Crit Care Med. 2013; 187(8): 823-831, doi: 10.1164/ rccm.201208-1518OC, indexed in Pubmed: 23392438.

32. Shteinberg M, Stein N, Adir Y, et al. Prevalence, risk factors and prognosis of nontuberculous mycobacterial infection among people with bronchiectasis: a population survey. Eur Respir J. 2018; 51(5), doi: 10.1183/13993003.02469-2017, indexed in Pubmed: 29545278.

33. Henkle E, Aksamit TR, Barker AF, et al. Pharmacotherapy for Non-Cystic Fibrosis Bronchiectasis: Results From an NTM Info \& Research Patient Survey and the Bronchiectasis and NTM Research Registry. Chest. 2017; 152(6): 1120-1127, doi: 10.1016/j.chest.2017.04.167, indexed in Pubmed: 28479113.

34. Axson EL, Bloom CI, Quint JK. Nontuberculous mycobacterial disease managed within UK primary care, 2006-2016. Eur J Clin Microbiol Infect Dis. 2018; 37(9): 1795-1803, doi: 10.1007/s10096-018-3315-6, indexed in Pubmed: 29951934.

35. Ito $\mathrm{Y}$, Hirai T, Fujita $\mathrm{K}$, et al. Increasing patients with pulmonary Mycobacterium avium complex disease and associated underlying diseases in Japan. J Infect Chemother. 2015; 21(5): 352-356, doi: 10.1016/j.jiac.2015.01.004, indexed in Pubmed: 25640532.

36. Park SW, Song JW, Shim TS, et al. Mycobacterial pulmonary infections in patients with idiopathic pulmonary fibrosis. J Korean Med Sci. 2012; 27(8): 896-900, doi: 10.3346/ jkms.2012.27.8.896, indexed in Pubmed: 22876056.

37. Brode SK, Jamieson FB, Ng R, et al. Increased risk of mycobacterial infections associated with anti-rheumatic medications. Thorax. 2015; 70(7): 677-682, doi: 10.1136/thoraxjnl-2014-206470, indexed in Pubmed: 25911222.

38. Słupek A, Zwolska Z, Miller M, et al. [Pulmonary mycobacteriosis--diagnostic problem and prevalence in Poland (a retrospective study)]. Pneumonol Alergol Pol. 1997; 65(5-6): 326-332, indexed in Pubmed: 9340058.

39. Wyrostkiewicz D, Skorupa W, Jakubowska L, et al. Mycobacterial lung disease in patients with cystic fibrosis - report of three cases. Pneumonol Alergol Pol. 2014; 82(6): 561-567, doi: 10.5603/PiAP.2014.0074, indexed in Pubmed: 25339567.

40. Adjemian J, Olivier KN, Prevots DR. Epidemiology of Pulmonary Nontuberculous Mycobacterial Sputum Positivity in Patients with Cystic Fibrosis in the United States, 2010-2014. Ann Am Thorac Soc. 2018; 15(7): 817-826, doi: 10.1513/AnnalsATS.201709-727OC, indexed in Pubmed: 29897781.

41. Gardner AI, McClenaghan E, Saint G, et al. Epidemiology of nontuberculous mycobacteria infection in children and young people with cystic fibrosis: analysis of UK Cystic Fibrosis Registry. Clin Infect Dis. 2018 [Epub ahead of print], doi: 10.1093/ cid/ciy531, indexed in Pubmed: 29982302. 